\title{
Molecular dynamics simulations of supercritical water confined within a carbon-slit pore
}

\author{
J. Martí, ${ }^{1, *}$ J. Sala, ${ }^{1}$ E. Guàrdia, ${ }^{1}$ and M. C. Gordillo ${ }^{2}$ \\ ${ }^{1}$ Departament de Física i Enginyeria Nuclear, Universitat Politècnica de Catalunya, B4-B5 Campus Nord, \\ 08034 Barcelona, Catalonia, Spain \\ ${ }^{2}$ Departamento de Sistemas Físicos, Químicos y Naturales, Facultad de Ciencias Experimentales, Universidad Pablo de Olavide, \\ Ctra. de Utrera, Km. 1. Sevilla, Spain \\ (Received 26 September 2008; published 20 March 2009)
}

\begin{abstract}
We report the results of a series of molecular dynamics simulations of water inside a carbon-slit pore at supercritical conditions. A range of densities corresponding from liquid $\left(0.66 \mathrm{~g} \mathrm{~cm}^{-3}\right)$ to gas environments $\left(0.08 \mathrm{~g} \mathrm{~cm}^{-3}\right)$ at the supercritical temperature of $673 \mathrm{~K}$ were considered. Our findings are compared with previous studies of liquid water confined in graphene nanochannels at ambient and high temperatures, and indicate that the microscopic structure of water evolves from hydrogen bond networks characteristic of hot dense liquids to looser arrangements where the dominant units are water monomers and dimers. Water permittivity was found to be very small at low densities, with a tendency to grow with density and to reach typical values of unconfined supercritical water at $0.66 \mathrm{~g} \mathrm{~cm}^{-3}$ ). In supercritical conditions, the residence time of water at interfaces is roughly similar to that of water in the central regions of the slabs, if the size of the considered region is taken into account. That time span is long enough to compute dynamical properties such as diffusion or spectral densities. Water diffusion in supercritical states is much faster at low densities, and it is produced in such a way that, at interfaces, translational diffusion is mainly produced along planes parallel to the carbon walls. Spectral frequency shifts depend on several factors, being temperature and density effects the most relevant. However, we can observe corrections due to confinement, important both at the graphene interface and in the central region of the water slab.
\end{abstract}

DOI: 10.1103/PhysRevE.79.031606 PACS number(s): 68.08.De, 61.30.Hn, 61.20.Qg, 31.15.xv

\section{INTRODUCTION}

Supercritical water (SCW) can change smoothly from a liquidlike fluid to a gaslike phase by adequately tuning the temperature and pressure of the sample, with properties that may differ significantly from those of water at ambient conditions. For instance, supercritical water is known to have an abnormally low dielectric constant and large diffusion coefficients [1], to mention only two particularly important examples. Due to such specificities, SCW is able to destroy organic wastes by oxidation (what is usually called supercritical water oxidation) [2], plays an important role in geochemistry (where it is a solvent for hydrothermal reactions) [3], and it can be used as a coolant in the so-called supercritical water nuclear reactors [4].

In most of the applications of SCW to technological uses, such as in the fields of chemistry and chemical engineering, it acts as a solvent or working fluid. However, it is known that experimental vessels are exposed to the high corrosivity produced by SCW [1]. Due to these difficulties, measurement setups are hard to prepare and perhaps for this reason the structure of SCW is hardly difficult to be measured and, consequently, it has become the subject of many a controversy [5-7]. Under supercritical conditions, hydrogen bonds (HBs) are very sensitive to temperature, density, and pressure changes. Nevertheless, to our knowledge, in a vast majority of experimental and theoretical works (including computer simulations) the existence of $\mathrm{HB}$ in SCW has been discovered in a wide range of thermodynamic conditions. So, a

\footnotetext{
*jordi.marti@upc.edu
}

significant amount of hydrogen bonding was detected by Matubayashi et al. [8] through NMR experiments and by Gorbaty and Kalinichev [9] from x-ray scattering measurements. Furthermore, computer simulations using a variety of potential models have reported the existence of HBs as well [10-12], usually from the existence of a minimum in the oxygen hydrogen radial distribution function $g_{\mathrm{OH}}(r)$, located around $2.4 \AA$ [12], very close to the equivalent position at ambient conditions. We should point out that in this context a very careful definition of $\mathrm{HB}$ is required, since definitions based on energetic criteria, which are commonly employed at room temperature, fail at SCW states due to its ambiguity [12].

Our contribution will present brand new computer simulations of supercritical water confined in a carbon-slit pore which has the form of a graphene slab periodically replicated. The study of water in carbon pores with graphitelike surfaces has been recently carried out by means of Monte Carlo $[13,17]$ and molecular dynamics (MD) simulations [14]. Carbon and silica pore confinement effects have recently been studied by Argyris et al. [15]. The present work is an extension of preliminary works where the same model system has been investigated at ambient $[18,19]$ and hightemperature subcritical conditions [20]. Structure and hydrogen bonding, as well as dielectric and dynamical properties of SCW confined in narrow graphene slabs at the nanoscale have been investigated by MD simulations. We will describe the methodology, models and details of the simulations in Sec. II, later on we will report and analyze the set of results in Sec. III and finally several conclusions will be drawn in Sec. IV. 
TABLE I. Characteristics of the MD simulations and effective charges of oxygens (in units of the electron charge).

\begin{tabular}{cccc}
\hline$T(\mathrm{~K})$ & $\rho\left(\mathrm{g} \mathrm{cm}^{-3}\right)$ & $N_{\text {water }}$ & $q_{\mathrm{O}}(e)$ \\
\hline 673 & 0.08 & 100 & -0.77 \\
673 & 0.16 & 200 & -0.79 \\
673 & 0.25 & 300 & -0.77 \\
673 & 0.33 & 400 & -0.77 \\
673 & 0.44 & 535 & -0.72 \\
673 & 0.55 & 670 & -0.72 \\
673 & 0.66 & 800 & -0.72 \\
633 & 0.526 & 550 & -0.78 \\
473 & 0.863 & 900 & -0.82 \\
298 & 1.0 & 1089 & -0.82 \\
\hline \hline
\end{tabular}

\section{MODELS AND SIMULATION DETAILS}

We considered $N$ water molecules located into two parallel graphene plates with no defects, forming a graphene slab. We set up a simulation box in the $x, y$, and $z$ directions with lengths of $34.4,34.1$, and $31 \AA$, respectively. We assumed the $z$ coordinate to be perpendicular to the graphene layers, and the usual periodic boundary conditions were considered only in the $x$ and $y$ directions. The temperature of the system was kept at $T=673 \mathrm{~K}$, slightly over the critical temperature of the water model employed in this work. The number of water molecules was set between $N=100$ and 800 , to cover the range of densities between $\rho=0.08$ and $\rho=0.66 \mathrm{~g} \mathrm{~cm}^{-3}$. Details of the MD simulations are presented in Table I.

Water-water inter-molecular and intramolecular interactions were modeled with a flexible simple point charged (SPC) potential which was specifically reparameterized to reproduce the main trends of the infrared spectrum of water at ambient conditions [21]. This flexible SPC potential has a critical point $\left(T_{c}=643 \mathrm{~K}, \rho_{c}=0.32 \mathrm{~g} \mathrm{~cm}^{-3}\right)$ which is close to the experimental one $\left(T_{c}=647 \mathrm{~K}, \rho_{c}=0.322 \mathrm{~g} \mathrm{~cm}^{-3}\right)$ [22]. Here we should point out that confinement may alter the location of a critical point and, even that the mere existence of such a critical point when a fluid is confined $[23,24]$ is unclear. As a possible reference for comparison, it should be noted that a hysteresis critical temperature of $543 \mathrm{~K}$ was obtained by Striolo et al. [25] for SPC/E water in a carbon-slit pore $1.6 \mathrm{~nm}$ wide, what means that, although hysteresis critical temperatures do not correspond to the pore critical temperature, confinement might produce the decreasing of critical point locations to lower values. Nevertheless, determining the critical point of our water model inside the carbon-slit pore is out of the scope of the present paper.

Water-carbon forces were assumed to be of the LennardJones type with the same parametrization employed in previous studies of water near carbon based surfaces, such as carbon nanotubes, graphite, or graphene slabs (see, for instance, Refs. [20,26-28]. It should be pointed out that effective charges of hydrogen and oxygen atoms corresponding to this particular potential model were parametererized to work at room temperature, leading to an averaged molecular dipole moment of water of $2.44 \mathrm{D}$ [29]. Conversely, low den- sity supercritical states are closer to gas systems than to dense liquids. Further, we should remember that the molecular dipole moment of water in gas phase is about $1.85 \mathrm{D}$. So, if MD simulations of SCW were conducted using the original effective charges, the total energy of the system would become too high, leading the system to collapse. The simplest way to handle this problem is by means of the systematic reduction of the molecular dipole moment of water. In our case, the final set of charges which guarantees configurational stability of modeled SCW states are reported in Table I. Note that the biggest reduction is about $12 \%$ in three cases of intermediate density. We can observe that the dependency between density (or temperature) and charge reduction is not monotonic. This suggests that a simultaneous dependence on both factors or, perhaps, even on additional variables (such as pressure) should be taken into account to establish a general rule for the optimization of the potential model.

To carry out the simulations we employed the integration algorithm of Berendsen et al. [30] with a time step of $0.5 \mathrm{fs}$ and a thermal bath coupling parameter of $10 \mathrm{fs}$. Short-range forces were truncated at half the box length and the Ewald summation technique as proposed by Spohr [31] was applied to account for the long-range Coulomb interactions. This particular modification consists in the enlargement of the $z$ coordinate of the simulation box by at least five times the $x$ and $y$ directions. Such three-dimensional (3D) Ewald procedure has been shown to be asymptotically equivalent to the $2 \mathrm{D}$ version, and appropriate to the simulation setup considered in the present work, but computationally less expensive. Each MD simulation run consisted of an initial equilibration period followed by production runs longer than $300 \mathrm{ps}$, in order to collect statistically meaningful properties. Initial runs (longer than $50 \mathrm{ps}$ in all cases) employed for equilibration purposes were discarded for the calculation of physical properties. In the calculation of the dielectric constants, the amount of statistics required was larger than $0.5 \mathrm{~ns}$.

\section{RESULTS}

During the MD simulations, we analyzed separately the properties of two different groups of water molecules as a function of their locations with respect to the confining walls: interfacial water is closer to a graphene plane, while bulklike water is further from the surface. The exact definition of these two groups comes from the corresponding oxygen density profiles (see below). These profiles are qualitatively similar to those recently reported by Cicero et al. [32] by means of first principle MD simulations at $400 \mathrm{~K}$ and by Brovchenko et al. [33] at temperatures around $600 \mathrm{~K}$.

\section{A. Structure and hydrogen bonding}

The usual way to present structural information of nonisotropic systems is by means of density profiles along a particular coordinate or geometrical order parameter. In the present case, we chose the $z$-axis coordinate as the relevant parameter and the corresponding density profiles for the oxygen water atoms are plotted in Fig. 1. There, we compare the 


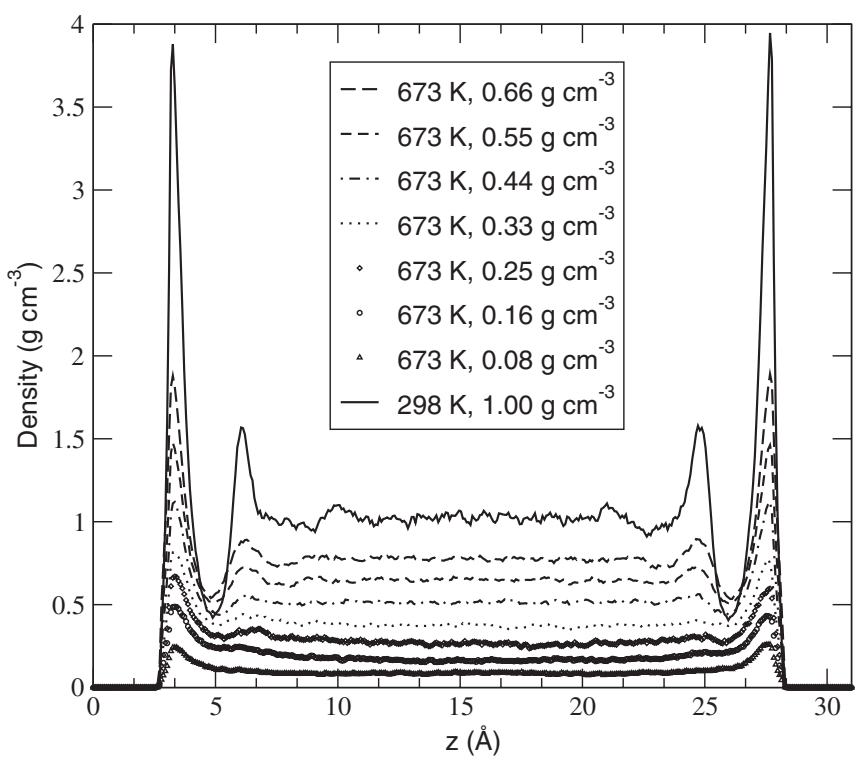

FIG. 1. Oxygen density profiles for supercritical and liquid water.

reference oxygen density profile at ambient conditions versus the whole set of profiles for confined SCW. A region of excluded volume $2.5 \AA$ wide is found at the two interfaces, in good agreement with Ref. [32]. At first sight, we observe that density is noticeably bigger at room temperature, both at interfaces and bulklike regions, decreasing gradually for the supercritical states. In addition, a third, less defined region seen at $298 \mathrm{~K}$ (what we called the "intermediate" region [18]) quickly vanishes when SCW is considered. Given these features, we assumed that molecules located at $z<5 \AA$ from slabs are interfacial water and those with $z \in[7.5,22.5] \AA$ are far enough from interfaces to be considered bulklike molecules. Both cutoff values are considered to be common to all thermodynamic states considered. Maxima related to the interfacial structure exist in all cases, although they become less well defined as the overall density of the system diminishes. Similar effects were observed for water at subcritical temperatures, in the range of 473-633 K [20]. Orientation of water molecules in the vicinity of the graphene sheets was found to be qualitatively equivalent to the case of subcritical states of water at high temperatures (see Figs. 3 and 4 of Ref. 20) and it is not shown for simplicity. We observed two relevant orientations corresponding to (1) water molecules with one hydrogen pointing to the surface (dangling hydrogen bonds) and (2) configurations where the instantaneous water plane is nearly parallel to the graphene sheet. However, those preferential orientations tend to be much less marked than at lower temperatures.

The hydrogen bond is surely the key feature that allows us to understand most of the peculiar properties of liquid water. It has been demonstrated $[10,12]$ that in computer simulations of SCW, the adequate choice to define $\mathrm{HB}$ is a purely geometrical definition or, at least, the combination of geometrical and energetical criteria, since the pure energetical criterion is based on an ambiguous choice of the water dimer configurational energy minimum. Consequently, as in previous studies of aqueous systems at supercritical conditions

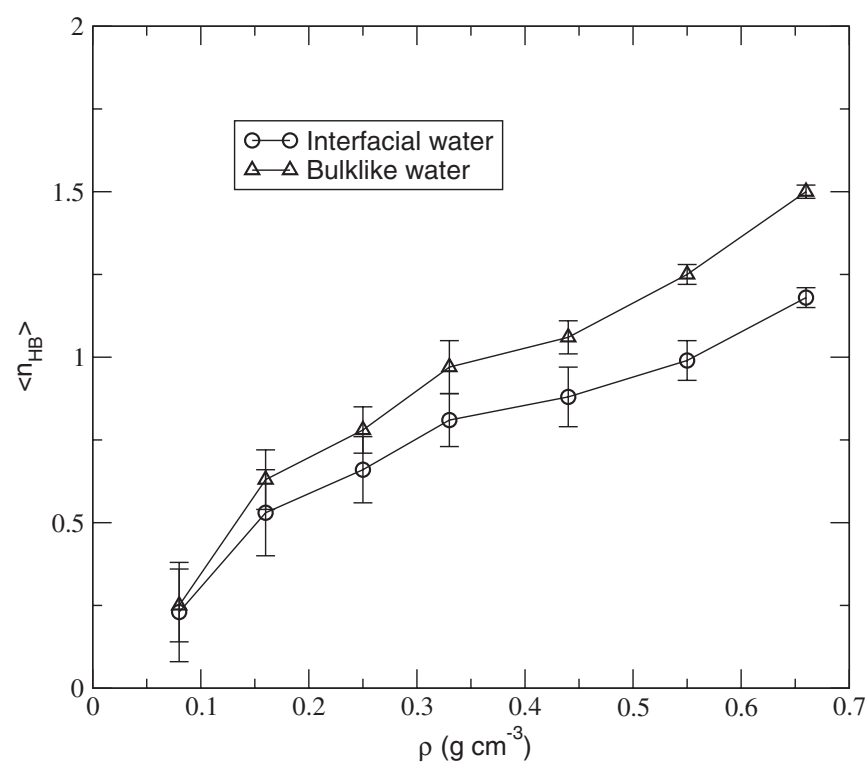

FIG. 2. Averaged hydrogen bond number for water in SCW at $673 \mathrm{~K}$ and in selected regions: interfaces and bulklike region.

$[12,34]$, we have assumed that two water molecules are $\mathrm{H}$-bonded if the next three geometrical conditions are fulfilled: (1) the distance between the oxygen atoms is smaller than $R_{\mathrm{OO}}=3.5 \AA$, (2) the distance between the "acceptor" oxygen and the "donor" hydrogen $R_{\mathrm{OH}}$ is smaller than $2.4 \AA$, (3) the $\mathrm{H}-\mathrm{O} \cdots \mathrm{O}$ angle is smaller than $30^{\circ}$.

The cutoff distances were obtained from the first minima of the corresponding radial distribution functions of bulk water [35], whereas the cutoff angle is set up in order to select linear HBs. A more detailed study where several classes of HBs were distinguished was reported by Boero et al. [36] but it is not considered here. The one we used is the standard procedure employed in a variety of computer simulations $[37,38]$. The averaged HB numbers $\left\langle n_{\mathrm{HB}}\right\rangle$ obtained in SCW are displayed in Fig. 2 and in Table II for the two relevant regions of the system (interface, bulklike). The results show a quasilinear dependence of $\left\langle n_{\mathrm{HB}}\right\rangle$ as a function of the mean density of the system for the two water types, although at low densities the values of $\left\langle n_{\mathrm{HB}}\right\rangle$ are very similar regardless of the location of water. However, at high densities the difference between the average $\mathrm{HB}$ number is about $25 \%(1.2$ for interfacial and 1.5 for bulklike water). These values indicate a microscopical structure made of a mixture of water dimers and trimers. However, when density is very low, namely below, $0.44 \mathrm{~g} \mathrm{~cm}^{-3}$ (see Fig. 2), the values of $\left\langle n_{\mathrm{HB}}\right\rangle$ fall below 1, what indicates that the dominant structures are basically monomers and dimers. Finally, in the lowest density case only a few dimers remain and we find out a majority of free water molecules, very close to the gaslike case.

In summary, our results indicate that, in SCW, the amount of hydrogen bonding at the interface formed by water with a hydrophobic wall is markedly smaller than at the bulklike central region at all densities. The dependence of $\left\langle n_{\mathrm{HB}}\right\rangle$ with the distance to the wall can be described by means of a quasi-linear dependence of rising values. This picture is radically different of that observed at hydrophiliclike interfaces [38] where the average number of HB tends to decrease as 
TABLE II. Occupation percentage, averaged HB number, static dielectric constant, and intermittent residence times of water for different thermodynamic states. $\varepsilon$ values suffer large fluctuations at the interfaces (see Ref. [20]).

\begin{tabular}{|c|c|c|c|c|c|c|}
\hline$T(\mathrm{~K})$ & $\rho\left(\mathrm{g} \mathrm{cm}^{-3}\right)$ & Region & Occupation \% & $\left\langle n_{\mathrm{HB}}\right\rangle$ & $\tau_{\text {res }}(\mathrm{ps})$ & $\varepsilon$ \\
\hline \multirow[t]{2}{*}{673} & 0.66 & Interfaces & 21 & 1.18 & 5.4 & 8.4 \\
\hline & & Bulklike & 79 & 1.50 & 74.6 & 4.5 \\
\hline \multirow[t]{2}{*}{673} & 0.55 & Interfaces & 21 & 0.99 & 4.6 & 6.6 \\
\hline & & Bulklike & 79 & 1.25 & 74.1 & 4.0 \\
\hline \multirow[t]{2}{*}{673} & 0.44 & Interfaces & 22 & 0.88 & 4.2 & 5.1 \\
\hline & & Bulklike & 78 & 1.06 & 73.5 & 3.4 \\
\hline \multirow[t]{2}{*}{673} & 0.33 & Interfaces & 22 & 0.81 & 3.7 & 4.4 \\
\hline & & Bulklike & 78 & 0.97 & 73.6 & 2.9 \\
\hline \multirow[t]{2}{*}{673} & 0.25 & Interfaces & 24 & 0.66 & 3.6 & 3.2 \\
\hline & & Bulklike & 76 & 0.78 & 67.9 & 2.2 \\
\hline \multirow[t]{2}{*}{673} & 0.16 & Interfaces & 26 & 0.53 & 3.7 & 2.5 \\
\hline & & Bulklike & 74 & 0.63 & 58.4 & 1.5 \\
\hline \multirow[t]{2}{*}{673} & 0.08 & Interfaces & 27 & 0.23 & 4.0 & 1.2 \\
\hline & & Bulklike & 73 & 0.25 & 52.1 & 0.7 \\
\hline \multirow[t]{2}{*}{633} & 0.526 & Interfaces & 20 & & 5.2 & 8.3 \\
\hline & & Bulklike & 80 & & 74.4 & 5.8 \\
\hline \multirow[t]{3}{*}{473} & 0.863 & Interfaces & 20 & & 16.0 & 34.0 \\
\hline & & Intermediate & 19 & & 7.5 & 19.0 \\
\hline & & Bulklike & 61 & & 58.0 & 30.0 \\
\hline \multirow[t]{3}{*}{298} & 1.0 & Interfaces & 23 & 3.20 & 54.0 & 172.0 \\
\hline & & Intermediate & 17 & 3.50 & 14.0 & 30.0 \\
\hline & & Bulklike & 60 & 3.55 & 127.0 & 35.0 \\
\hline
\end{tabular}

the distance to interface rises, result that has been attributed to the hydrophilic effect.

\section{B. Static dielectric constant}

The relative dielectric permittivity (static dielectric constant) $\varepsilon$ is the usual way to describe the dielectric characteristics of a liquid. In the case of a system with long-range interactions treated by the Ewald method with conducting boundary conditions, $\varepsilon$ is given by $[39,40]$

$$
\varepsilon=1+\frac{4 \pi N\left\langle\mu^{2}\right\rangle G_{k}}{3 V k_{B} T},
$$

where $N$ is the number of water molecules, $V$ is the accessible volume, $\vec{\mu}(t)$ is the molecular dipole moment of water, $k_{B}$ is the Boltzmann constant, and $G_{k}$ is the finite system Kirkwood $g$ factor:

$$
G_{k}=\frac{\left\langle M^{2}\right\rangle}{N\left\langle\mu^{2}\right\rangle},
$$

with $\vec{M}(t)=\sum_{i=1}^{N} \vec{\mu}_{i}(t)$ being the total dipole moment of the system. The brackets indicate statistical average over independent configurations.

The results obtained for SCW are included in Table II, where they are compared to lower temperature values and are also presented in Fig. 3 for clarity purposes. In all cases, $\varepsilon$ values are in the range of the ones corresponding to SCW states $[1,41]$ and are always larger in the interfacial than in the central region. This feature was already observed at ambient conditions and at high subcritical temperatures [20] and it agrees qualitatively well with the findings of Ballenegger

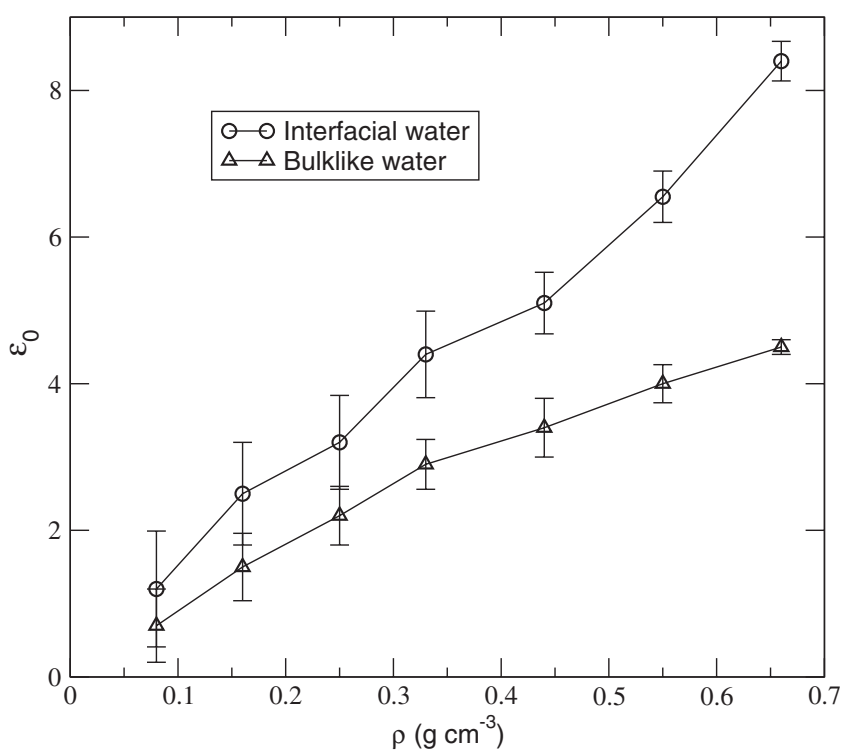

FIG. 3. Static dielectric constant of water in SCW at $673 \mathrm{~K}$ and in selected regions: interfaces and bulklike region. 
and Hansen [42] for slab and spherical geometries, who observed the general increase of the dielectric constant when moving from bulklike regions to the interfaces. It should be pointed out that permittivity tends to be very small (of the order of 1) at the lowest density considered $\left(0.08 \mathrm{~g} \mathrm{~cm}^{-3}\right)$ because we are very close to gaslike characteristics and the structure, as described above, is basically the same for water molecules belonging to the interfaces and to the central region. When density rises, structure tends to be progressively more complex, including trimers and short water chains, which leads to different values for $\varepsilon$ in both zones. So, at the highest SCW density $\left(0.66 \mathrm{~g} \mathrm{~cm}^{-3}\right)$ permittivity in the interfaces is roughly twice bigger than in the bulklike region. This fact may be attributed, on the one hand, to a larger extent of molecular orientational order [18], and on the other hand, to the observed large fluctuations of the electrostatic potential in the water layer nearest to the interfaces. Such fluctuations producing large changes in the dipolar moment of water are a consequence of the influence of changes in the hydrogen bonding when water is located in the vicinity of the carbon walls.

\section{Residence time of water molecules}

Residence time of a water molecule in a given region is defined as the mean time that the molecule spends in such region before moving away. Two classes of those times $\tau_{\text {res }}^{I}$ and $\tau_{\text {res }}^{C}$ were first defined by Impey et al. [43]. The $I$ superscript indicates the "intermittent" character of the computed time: multiple reentrance of water molecules until they definitely leave the given region for a period of time of infinite length is allowed. For practical purposes, such "infinite" length in time is taken to be of the order of picoseconds. Conversely, the $C$ superscript indicates the "continuous" residence time, i.e., it is computed until a water molecule leaves the region for the first time. These times can be considered as corresponding to "stationary" (intermittent) and to "transient" (continuous) regimes of persistence of water in a given region, respectively. Since the residence time that is usually measured experimentally is the intermittent one, we will analyze its behavior in SCW through the results reported in Fig. 4. The corresponding numerical values have been also included in Table II.

We collected the values of $\tau_{\text {res }}^{I}$ for water in the interfacial and bulklike regions. From a general point of view, these are raw numerical values likely to be directly compared to experimental measurements. We first note that values for bulklike water are about 15-20 times larger than those of interfacial water in SCW. These differences are strikingly bigger than those observed at subcritical states for the model employed here [20], where $\tau_{\text {res }}^{I}$ in bulklike water is about 3-4 times that of interfacial water. It should be taken into account that the characteristics of hydrogen bonding at the interface are radically different of those at the central part of the system and that the $z$ span of each region is very different in size. So, assuming that water molecules roam evenly the assigned volumes, we can "normalize" $\tau_{\text {res }}^{I}$ with the width of the considered zone and obtain a parameter basically independent of the size of the water slab. In such a case, the

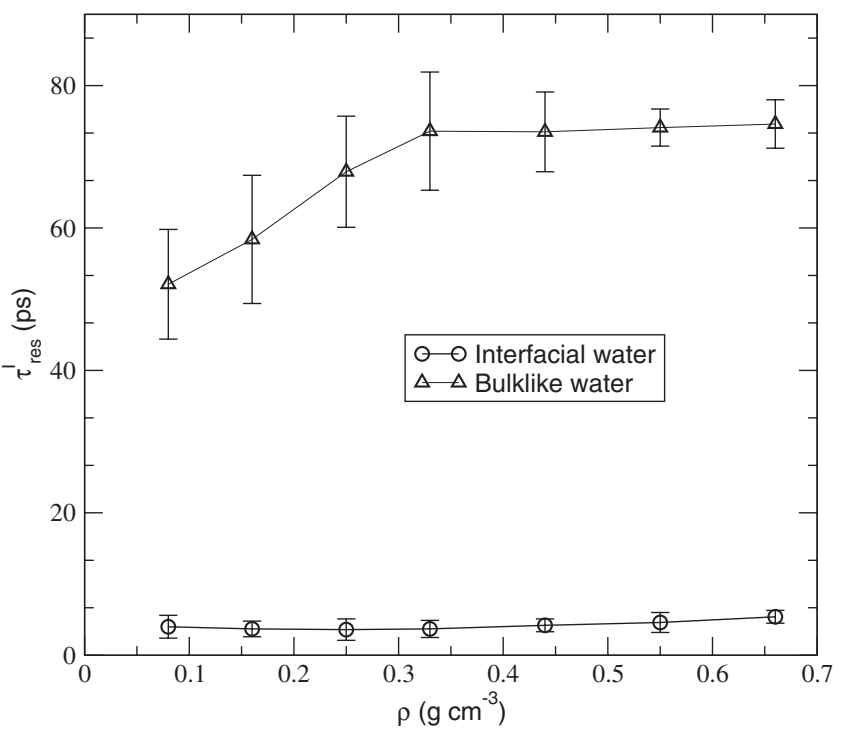

FIG. 4. Intermittent residence time of water molecules in SCW at $673 \mathrm{~K}$ and in selected regions: interfaces and bulklike region.

"normalized residence times" at the interface and in the central bulklike region are of about 24 and 8 ps, respectively, at ambient conditions [18] whereas they change to much smaller values for SCW, of around 2 ps at the interfacial regions (width of $2.2 \AA$ for all states) and between $2.5-3.5 \mathrm{ps}$ in the bulklike regions (width of $16 \AA$ at $298 \mathrm{~K}$ and of $21 \AA$ at SCW).

Summarizing, such normalized residence times of water at interfaces are higher than those in bulk at subcritical conditions, whereas they tend to decrease at higher temperatures. In low density SCW, we observe that water tends to spend very similar times at the two regions, of the order of 2 ps. A possible reason for this convergence is the fact that the high thermal energy involved in SCW states produces a large mobility of water, that leads to quick exchanges between the selected regions. Here the hydrophobic effect seems to be less important than the thermal one. When silica pores are considered, the dependence of water residence times on the nature of the silica surface is more important than the one found in the present work [15]. Experimental findings of Tassaing and Bellissent-Funel [16] reported values much smaller for "residence" times obtained from fits of the quasielastic incoherent neutron scattering spectra, of the order of $0.1-0.2$ ps. These experimental times were measured for unconstrained water and follow from a jump diffusion model, so they could be related with the time spent by a water molecule to diffuse away from the cage formed by their nearest neighbors. Conversely, in our case the "residence" times have been computed as the time spent by water molecules either in the interfacial or bulklike region. Finally, the fact that water molecules remain in each region for 2 ps or more in all cases, ensures the meaningfulness of the calculation of other dynamical quantities such as transport coefficients and spectral densities, which we will address in the next section.

\section{Dynamics: Translational diffusion}

It is well known that water self-diffusion coefficients suffer considerable variations when the liquid is under confine- 


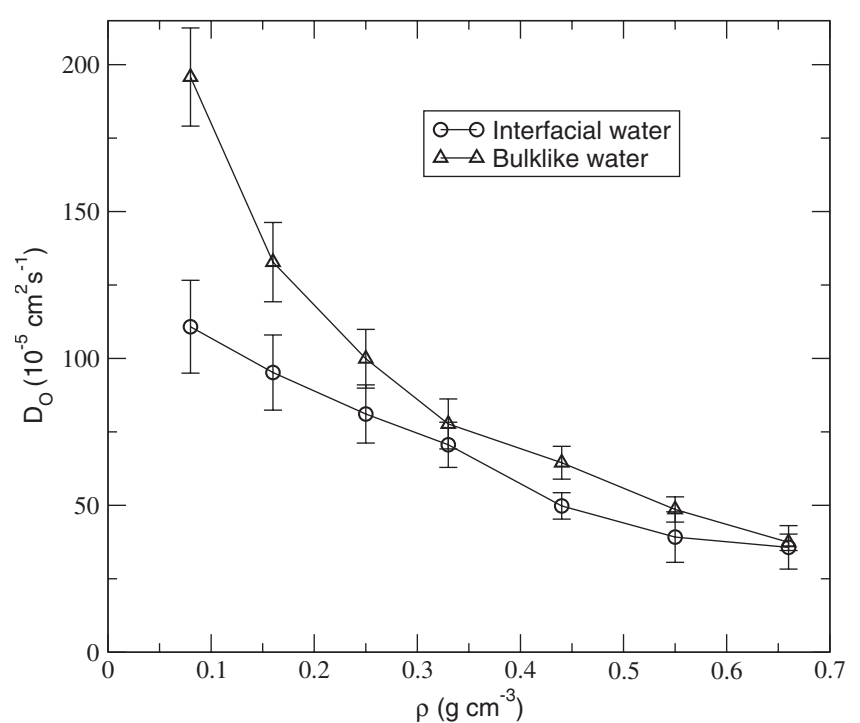

FIG. 5. Selfdiffusion coefficients of oxygens in SCW at $673 \mathrm{~K}$ and in selected regions: interfaces and bulklike region.

ment with respect to the unconstrained bulk water values [44]. The changes depend basically on the geometry and characteristics of the confining media and are different for interfacial and bulklike areas [19]. In this work, we present results from calculations of the self-diffusion coefficients $D$ of water for both interfacial and bulklike regions. We have considered the diffusion coefficients only for oxygen atoms $\left(D_{\mathrm{O}}\right)$, because the center-of-mass of the water molecule is roughly located on the oxygen site. The $D_{\mathrm{O}}$ were calculated by the time integration of the oxygen velocity autocorrelation functions.

The results are presented in Fig. 5 for SCW at different densities. There, we can see that water at low densities diffuses much faster than in closely packed systems, as expected. This is in good agreement with the neutron scattering data of Tassaing and Bellissent-Funel [16], both concerning the reported values of $D$ and the observed tendency to decrease with increasing densities. Not surprisingly, interfacial water has lower $D_{\mathrm{O}}$ than water located in the central part of the system, as it was observed in MD simulations at lower temperatures performed with the same potential model employed here [20]. The interpretation of such behavior was based on the fact that diffusion in water within slabs is mainly due to molecular motions along planes parallel to the surfaces, since diffusion coefficients of interfacial water computed separately for the parallel $(X Y)$ and perpendicular to the surface $(Z)$ directions revealed $D_{X Y}>D_{Z}$ at temperatures up to $400 \mathrm{~K}[19,45]$. The dependence of $D_{\mathrm{O}}$ on density is somewhat similar for the two water types: diffusion of $\mathrm{SCW}$ at interfaces tends to decrease in a smoothly exponential way, whereas $D_{\mathrm{O}}$ of water in the central slab region decreases in a faster exponential regime, passing from $D_{\mathrm{O}}$ $\sim 200 \times 10^{-5} \mathrm{~cm}^{2} / \mathrm{s}$ at $0.08 \mathrm{~g} \mathrm{~cm}^{-3}$ to $\sim 35 \times 10^{-5} \mathrm{~cm}^{2} / \mathrm{s}$ at $0.66 \mathrm{~g} \mathrm{~cm}^{-3}$.

Concerning the effect of confinement, it should be noted that the self-diffusion coefficient of the oxygen atoms in unconstrained liquid water at $673 \mathrm{~K}$ is about 39.4 $\times 10^{-5} \mathrm{~cm}^{2} / \mathrm{s}$ for this particular potential model [12], in

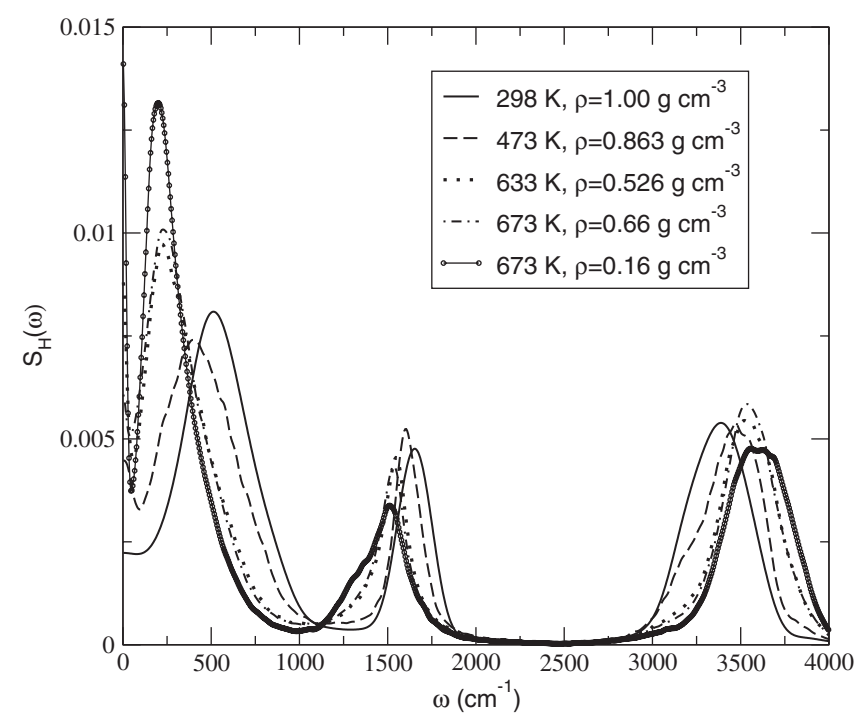

FIG. 6. Spectral densities of hydrogen atoms at several thermodynamic conditions: comparison between supercritical and liquid water.

qualitative good agreement with the experimental findings of Lamb et al. [46] obtained by means of NMR spin echo techniques. So, confinement of SCW at high density tends to reduce slightly water diffusion, regardless of the location of the water molecules in the system (either interfaces or central region).

\section{E. Dynamics: Molecular spectroscopy}

Molecular spectroscopy is a powerful tool able to shed light into the microscopic dynamics of liquids. Among the different experimental methods, infrared and Raman spectroscopy are two of the most suitable techniques, able to explore a wide frequency range between 0 and $4000 \mathrm{~cm}^{-1}$ $[47,48]$. In this work we will present spectral densities of hydrogen atoms $S_{\mathrm{H}}(\omega)$ for water in different environments and in variable thermodynamic conditions. Some features observed in $S_{\mathrm{H}}(\omega)$ (frequency maxima locations) can be directly compared with infrared or Raman data [49]. The calculation of $S_{\mathrm{H}}(\omega)$ was done by Fourier transforming the hydrogen velocity autocorrelation functions $\left\langle\vec{v}_{\mathrm{H}}(t) \cdot \vec{v}_{\mathrm{H}}(0)\right\rangle$, where the average is done over a set of configurations at different times along an equilibrated trajectory.

As it can be seen from Fig. 6, the full spectral range considered shows three well defined parts: librational motions $\left(100-1000 \mathrm{~cm}^{-1}\right)$, bending vibrations (1200-2000 $\mathrm{cm}^{-1}$ ) and $\mathrm{OH}$ stretching vibrations $\left(3000-4000 \mathrm{~cm}^{-1}\right)$. Let us describe each part in detail. Figure 6 is devoted to analyzing the influence of temperature on frequency shifts and, in a more detailed scale, to distinguish between low and high density SCW states. In the region of librational motions the main feature observed is a red shift of the main band (centered around $500 \mathrm{~cm}^{-1}$ at ambient conditions) towards lower values, of the order of $200 \mathrm{~cm}^{-1}$. Intermediate temperatures such as $473 \mathrm{~K}$ show the same maximum located around $400 \mathrm{~cm}^{-1}$. When we move to higher temperatures and towards SCW states, this frequency maximum shifts back- 


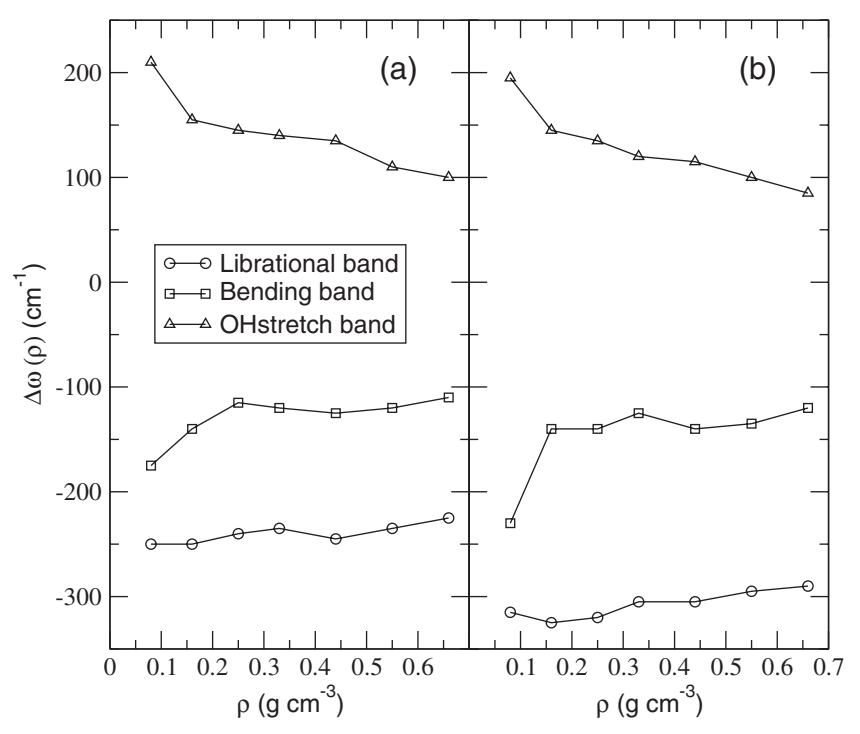

FIG. 7. Maxima frequency shifts $\Delta \omega(\rho)$ for spectral densities of hydrogens in SCW at $673 \mathrm{~K}$ and in selected frequency regions: librational, bending, $\mathrm{OH}$ stretch. (a) Values computed for water molecules located at the interfaces; (b) Values computed for water molecules located at the bulklike region.

wards until 200-230 $\mathrm{cm}^{-1}$. A band maximum around $240 \mathrm{~cm}^{-1}$ (i.e., $30 \mathrm{meV}$ ) was reported by Tassaing and Bellissent-Funel [16] from their neutron scattering experiments, for bulk SCW at $653 \mathrm{~K}$ and a density of $0.59 \mathrm{~g} / \mathrm{cm}^{-3}$. Hence, our results are in good qualitative agreement with these experiments and indicate that influence of confinement plays a minor role when compared to the thermodynamical effect of moving from ambient to supercritical states.

In our opinion, this particular redshift of the librational band can be attributed to a double contribution: (1) the temperature effect and (2) the confinement effect. In first place, we recently observed [45] that in bulk unconstrained water, the increase of temperature favors the displacement of librational frequency maxima to lower values. Secondly, in strongly packed systems, such as in water in carbon nanotubes [50] or in water in very narrow graphene channels [45] (separated less than $1 \mathrm{~nm}$ ), confinement favors redshifts of rotational bands, although to a lesser extent than it does the temperature rise. In addition, it should be mentioned that when the two cases of $T=673 \mathrm{~K}$ are compared, we observe that the biggest frequency shift corresponds to a density of $0.16 \mathrm{~g} \mathrm{~cm}^{-3}$ and is located around only $200 \mathrm{~cm}^{-1}$, i.e., in the region of intermolecular vibrations in water. This suggests that gaslike structures (dominant at low densities and SCW states) implicitly produce low frequency librational motions. In order to study the density influence with a deeper detail, we reported the relative frequency shifts $\Delta \omega(\rho)$ for the three relevant bands as a function of density in Fig. 7, separating the cases of interfacial and bulklike water. The magnitude of a spectral shift has been defined for each density $\rho$ as

$$
\Delta \omega(\rho) \equiv \omega(\rho)-\omega_{\text {ref }},
$$

where the "reference" $\left(\omega_{\text {ref }}\right)$ is the value of frequency maximum at $298 \mathrm{~K}$ and $1 \mathrm{~g} \mathrm{~cm}^{-3}$ (see Table III). This leads to positive (negative) values for blue shifts (red shifts). The magnitude of $\Delta \omega(\rho)$ is between 200-400 wave numbers for the librational region, what means that rotational motions are the most affected by frequency changes when the system is heated from room to supercritical temperatures. Interestingly, the shape of $\Delta \omega(\rho)$ is very similar for interfacial and bulklike water, being the absolute value of the latter somewhat bigger. Hopefully these shifts can be experimentally measured and compared to the present data.

In the bending region, the most relevant feature (Fig. 6) is a redshift of about $140 \mathrm{~cm}^{-1}$ between ambient and low density SCW conditions, with intermediate values of the bending peak diminishing with rising temperatures and decreasing densities. At the light of Fig. 7, the values of $\Delta \omega(\rho)$ are moderately lower than in the librational case, now in the range of 100-200 wave numbers, although a marked difference between interfacial and bulklike water arises at the lowest density: at $0.08 \mathrm{~g} \mathrm{~cm}^{-3}$ a larger redshift is recorded when water is located in the central region of slabs. This might be due to the strong gaslike characteristics of such state and to the fact that bending frequencies in gases are lower than those of the condensed liquid phases [51]. Further, in the bending region of the infrared spectrum for SCW at low densities obtained by infrared absorption spectroscopy [52], redshifts of about $30-40 \mathrm{~cm}^{-1}$ have been observed. This means that the flexible SPC model employed in the present work tends to overemphasize the magnitude of frequency shifts associated with bending vibrations.

The last frequency range to be analyzed is the $\mathrm{OH}$ stretching region. There we find a very different behavior, with blueshifts in all cases (Fig. 6), the most important corresponding to the case of SCW at low density. Blueshifts of similar magnitude were measured using neutron scattering techniques by Ricci et al. [53] in unconstrained SCW at $673 \mathrm{~K}$ at the density of $0.66 \mathrm{~g} \mathrm{~cm}^{-3}$. Tassaing et al. [52] published a study of a complete set of thermodynamical states including SCW, obtained by infrared spectroscopy, where frequency shifts towards the blue of similar magnitude than those presented in Figs. 6 and 7 were reported. It has to be pointed out that all experimental data were obtained for liquid and supercritical unconstrained water sets. When confinement is considered, Gilijamse et al. [54] reported spectra of water dissolved in acetone and $\mathrm{CCl}_{4}$ measured by femtosecond midinfrared pump-probe experiments, and showed that for such a system, the $\mathrm{OH}$-stretching main band was blueshifted about $150 \mathrm{~cm}^{-1}$. This magnitude for the frequency shift is quite close to the values obtained in the present work. From the computational side, Tummala and Striolo [55] recently reported very similar values and signs of $\Delta \omega$ when considering the same water-acetone- $\mathrm{CCl}_{4}$ mixture than Gilijamse et al. [54].

These findings are not surprising at all, since the analyzed states present microscopic structures close to those of gases or water steam. As we pointed out above, gaslike systems have vibrational frequencies significantly different to those of liquid ones being in the case of $\mathrm{OH}$ stretch in the range of 3700 wave numbers [51]. A closer look by means of the findings of Fig. 7 indicates a very similar profile of $\Delta \omega(\rho)$ when interfacial and bulklike water are compared. The common feature is a tendency to smaller frequency shifts at the 
TABLE III. Band maxima of spectral densities of hydrogens in water for relevant regions (interfaces, bulklike). Estimated uncertainties are about $0.5-1 \%$ for each value.

\begin{tabular}{|c|c|c|c|c|c|}
\hline $\mathrm{T}(\mathrm{K})$ & $\rho\left(\mathrm{g} \mathrm{cm}^{-3}\right)$ & Region & Librational $\left(\mathrm{cm}^{-1}\right)$ & Bending $\left(\mathrm{cm}^{-1}\right)$ & $\mathrm{OH}$ stretch $\left(\mathrm{cm}^{-1}\right)$ \\
\hline \multirow[t]{2}{*}{673} & 0.08 & Interfaces & 205 & 1460 & 3660 \\
\hline & & Bulklike & 205 & 1425 & 3645 \\
\hline \multirow[t]{2}{*}{673} & 0.16 & Interfaces & 205 & 1495 & 3605 \\
\hline & & Bulklike & 195 & 1515 & 3605 \\
\hline \multirow[t]{2}{*}{673} & 0.25 & Interfaces & 215 & 1520 & 3595 \\
\hline & & Bulklike & 200 & 1515 & 3585 \\
\hline \multirow[t]{2}{*}{673} & 0.33 & Interfaces & 220 & 1515 & 3590 \\
\hline & & Bulklike & 215 & 1530 & 3570 \\
\hline \multirow[t]{2}{*}{673} & 0.44 & Interfaces & 210 & 1510 & 3585 \\
\hline & & Bulklike & 215 & 1515 & 3565 \\
\hline \multirow[t]{2}{*}{673} & 0.55 & Interfaces & 220 & 1515 & 3560 \\
\hline & & Bulklike & 225 & 1520 & 3550 \\
\hline \multirow[t]{2}{*}{673} & 0.66 & Interfaces & 230 & 1525 & 3550 \\
\hline & & Bulklike & 230 & 1535 & 3535 \\
\hline \multirow[t]{2}{*}{633} & 0.526 & Interfaces & 225 & 1525 & 3620 \\
\hline & & Bulklike & 250 & 1535 & 3550 \\
\hline \multirow[t]{2}{*}{473} & 0.863 & Interfaces & 245 & 1595 & 3580 \\
\hline & & Bulklike & 405 & 1615 & 3475 \\
\hline \multirow[t]{2}{*}{298} & 1.0 & Interfaces & 455 & 1635 & 3450 \\
\hline & & Bulklike & 520 & 1655 & 3370 \\
\hline
\end{tabular}

highest densities. This is in agreement with the explanation given above in terms of the comparison of confined SCW with dense liquids and gases. This tendency to blueshifting is difficult to explain using simple arguments, but we tend to believe that two factors are playing a role: on the one hand, the size of the interfacial region and, on the other hand, the interactions with the walls and the surroundings (internal water).

\section{CONCLUDING REMARKS}

Results from computer simulations of SCW confined in slabs limited by graphene walls have been presented and analyzed. We have performed a series of MD simulations of SCW modeled through a flexible SPC potential, initially set up to fairly reproduce structure and dynamics of water at ambient conditions, adequately modified to model supercritical water states in confinement conditions. This has been done by means of subtle modifications of the molecular dipole of water in the SCW states. In all cases, water-carbon interactions have been modeled by Lennard-Jones forces. Once a set of equilibrated SCW configurations was generated, we analyzed structure including hydrogen bonding, permittivity, and dynamical aspects of water.

Water molecules in SCW can be divided into two groups: (1) those pertaining to a central region between 7.5 and
$22.5 \AA$, containing about $80 \%$ of all molecules (in all SCW cases), which we call bulklike water and (2) water located nearby the two interfaces (i.e., those with its $z$ coordinate less than $5 \AA$ away from the graphene sheets), which are about $20 \%$ of all molecules and that we called interfacial water (see Table III). Density profiles show that interfacial regions exist at all densities, with a tendency to disappear at low densities, leading to systems with a greater degree of homogeneity. Hydrogen bonding in constrained SCW is dramatically reduced at low densities, with monomers and some remaining dimers as dominant structures. This effect is seen for both interfacial and bulklike water, whereas static dielectric constants are in the normal range in bulk SCW (about 5-10) but showing a clear tendency to decrease at low densities. At the lowest density considered $\left(0.08 \mathrm{~g} \mathrm{~cm}^{-3}\right)$, permittivity converges to values as low as $\varepsilon \sim 1$ in the two relevant regions.

We have computed the residence time of water at interfaces and in the central part of the system. Our results indicate that, when we consider the size of each part, the intermittent times of water in the two selected regions are roughly equivalent in SCW and that they are long enough to study some relevant dynamical properties. So, computed oxygen self-diffusion coefficients in supercritical states are much larger at low densities than those at high density states, in the same way as it happens in bulk unconstrained systems $[12,46]$. Diffusion of water at interfaces has been observed to happen due to motion of water along planes parallel to the graphene walls. Spectral densities for the hydrogen atoms have been computed in order to find out the relevant 
frequency maxima and shifts of water at the midinfrared region, distinguishing between the contribution of interfacial and bulklike water. We observed redshifts of frequency maxima respect to the reference maxima (those of hydrogen at ambient conditions) in the librational and bending regions, whereas blue shifts appear in the $\mathrm{OH}$-stretch region. These frequency shifts depend of several factors, being temperature, density and confinement effects the most important.

\section{ACKNOWLEDGMENTS}

We gratefully acknowledge financial support from the "Direcció General de Recerca" of the Generalitat de Catalunya (Grant No. 2005SGR-00779), the "Ministerio de Educación y Ciencia" (MEC) of Spain (Grants No. FIS200612436-C02-01 and FIS2006-02356), from European Union FEDER funds (UNPC-E015) to the Junta de Andalucia for financial support under Grant No. P06-FQM-01869.
[1] H. Weingärtner and E. U. Franck, Angew. Chem., Int. Ed. 44, 2672 (2005).

[2] R. W. Shaw, T. B. Brill, A. A. Clifford, C. A. Eckert, and E. U. Franck, Chem. Eng. News 69, 26 (1991).

[3] D. T. Rickard and F. E. Wickman, Chemistry and Geochemistry of Solutions at High Temperatures and High Pressures (Pergamon, Oxford, 1981).

[4] G. S. Was, P. Ampornrat, G. Gupta, S. Teysseyre, E. A. West, T. R. Allen, K. Sridharan, L. Tan, Y. Chen, X. Ren, and C. Pister, J. Nucl. Mater. 371, 176 (2007).

[5] T. Tassaing, M.-C. Bellissent-Funel, B. Guillot, and Y. Guissani, Europhys. Lett. 42, 265 (1997).

[6] A. Botti, F. Bruni, M. A. Ricci, and A. K. Soper, J. Chem. Phys. 109, 3180 (1998).

[7] A. A. Chialvo and P. T. Cummings, Adv. Chem. Phys. 109, 115 (1999), and references therein.

[8] N. Matubayashi, C. Wakai, and M. Nakahara, J. Chem. Phys. 107, 9133 (1997).

[9] Y. E. Gorbaty and A. G. Kalinichev, J. Phys. Chem. 99, 5336 (1995).

[10] A. G. Kalinichev and J. D. Bass, J. Phys. Chem. A 101, 9720 (1997).

[11] A. Famulari, R. Specchio, M. Sironi, and M. Raimondi, J. Chem. Phys. 108, 3296 (1998).

[12] J. Martí, J. Chem. Phys. 110, 6876 (1999).

[13] J. C. Liu and P. A. Monson, Langmuir 21, 10219 (2005).

[14] Q. Cai, M. J. Biggs, and M. A. Seaton, Phys. Chem. Chem. Phys. 10, 2519 (2008).

[15] D. Argyris, N. R. Tummala, A. Striolo, and D. R. Cole, J. Phys. Chem. C 112, 13587 (2008).

[16] T. Tassaing and M.-C. Bellissent-Funel, J. Chem. Phys. 113, 3332 (2000).

[17] A. Wongkoblap and D. D. Do, Mol. Phys. 106, 627 (2008).

[18] J. Martí, G. Nagy, M. C. Gordillo, and E. Guàrdia, J. Chem. Phys. 124, 094703 (2006).

[19] J. Martí, G. Nagy, M. C. Gordillo, and E. Guàrdia, J. Phys. Chem. B 110, 23987 (2006).

[20] G. Nagy, M. C. Gordillo, E. Guàrdia, and J. Martí, J. Phys. Chem. B 111, 12524 (2007).

[21] J. Martí, J. A. Padró, and E. Guàrdia, J. Mol. Liq. 62, 17 (1994).

[22] C. C. Liew, H. Inomata, and K. Arai, Fluid Phase Equilib. 144, 287 (1998).

[23] R. Evans, U. Marini Bettolo Marconi, and P. Tarazona, J. Chem. Phys. 84, 2376 (1986).

[24] P. C. Ball and R. Evans, Langmuir 5, 714 (1989).
[25] A. Striolo, K. E. Gubbins, M. S. Gruszkiewicz, D. R. Cole, J. M. Simonson, A. A. Chialvo, P. T. Cummings, T. D. Burchell, and K. L. More, Langmuir 21, 9457 (2005).

[26] M. C. Gordillo and J. Martí, Chem. Phys. Lett. 329, 341 (2000).

[27] J. Martí and M. C. Gordillo, J. Chem. Phys. 119, 12540 (2003).

[28] M. C. Gordillo, G. Nagy, and J. Martí, J. Chem. Phys. 123, 054707 (2005).

[29] J. Martí, Phys. Rev. E 61, 449 (2000).

[30] H. J. C. Berendsen, J. P. M. Postma, W. F. van Gunsteren, A. DiNola, and J. R. Haak, J. Chem. Phys. 81, 3684 (1984).

[31] E. Spohr, J. Chem. Phys. 107, 6342 (1997).

[32] G. Cicero, J. G. Grossman, E. Schwegler, F. Gygi, and G. Galli, J. Am. Chem. Soc. 130, 1871 (2008).

[33] I. Brovchenko, A. Geiger, and A. Oleinikova, J. Phys.: Condens. Matter 16, S5345 (2004).

[34] E. Guàrdia, D. Laria, and J. Martí, J. Phys. Chem. B 110, 6332 (2006).

[35] J. Martí, J. A. Padró, and E. Guàrdia, J. Chem. Phys. 105, 639 (1996).

[36] M. Boero, K. Terakura, T. Ikeshoji, C. C. Liew, and M. Parrinello, Phys. Rev. Lett. 85, 3245 (2000).

[37] A. Luzar and D. Chandler, J. Chem. Phys. 98, 8160 (1993).

[38] S. R. P. da Rocha, K. P. Johnston, R. E. Westacott, and P. J. Rossky, J. Phys. Chem. B 105, 12092 (2001).

[39] S. W. de Leeuw, J. W. Perram, and E. R. Smith, Proc. R. Soc. London, Ser. A 388, 177 (1983).

[40] E. Guàrdia and J. Martí, Phys. Rev. E 69, 011502 (2004).

[41] S. Deguchi and K. Tsujii, Soft Matter 3, 797 (2007).

[42] V. Ballenegger and J.-P. Hansen, J. Chem. Phys. 122, 114711 (2005).

[43] R. M. Impey, P. A. Madden, and I. R. McDonald, J. Phys. Chem. 87, 5071 (1983).

[44] N. Choudhury and B. M. Pettitt, J. Phys. Chem. B 109, 6422 (2005).

[45] M. C. Gordillo and J. Martí, Phys. Rev. B 75, 085406 (2007).

[46] W. J. Lamb, G. A. Hoffman, and J. Jonas, J. Chem. Phys. 74, 6875 (1981).

[47] D. Eisenberg and W. Kauzmann, The Structure and Properties of Water (Clarendon Press, Oxford, 1969).

[48] G. E. Walrafen, in Water: A Comprehensive Treatise, edited by F. Franks (Plenum Press, New York, 1972), Vol. 1.

[49] J. Martí, E. Guàrdia, and J. A. Padró, J. Chem. Phys. 101, 10883 (1994).

[50] J. Martí and M. C. Gordillo, Phys. Rev. B 63, 165430 (2001). 
[51] S. H. Chen, K. Toukan, C. K. Loong, D. L. Price, and J. Teixeira, Phys. Rev. Lett. 53, 1360 (1984).

[52] T. Tassaing, Y. Danten, and M. Besnard, J. Mol. Liq. 101, 149 (2002).

[53] M. A. Ricci, M. Nardone, A. Fontana, C. Andreani, and W.
Hahn, J. Chem. Phys. 108, 450 (1998).

[54] J. J. Gilijamse, A. J. Lock, and H. J. Bakker, Proc. Natl. Acad. Sci. U.S.A. 102, 3202 (2005).

[55] N. R. Tummala and A. Striolo, J. Phys. Chem. B 112, 10675 (2008). 